\title{
Improving spatial resolution for EIT reconstructed images through measurement strategies
}

DOI:

10.1109/ICSIPA.2013.6707968

Link to publication record in Manchester Research Explorer

\section{Citation for published version (APA):}

Chin, R. K. Y., \& York, T. A. (2013). Improving spatial resolution for EIT reconstructed images through measurement strategies. In IEEE ICSIPA 2013 - IEEE International Conference on Signal and Image Processing Applications/IEEE ICSIPA - IEEE Int. Conf. Signal Image Process. Appl. (pp. 5-10). IEEE Computer Society . https://doi.org/10.1109/ICSIPA.2013.6707968

Published in:

IEEE ICSIPA 2013 - IEEE International Conference on Signal and Image Processing Applications|IEEE ICSIPA IEEE Int. Conf. Signal Image Process. Appl.

\section{Citing this paper}

Please note that where the full-text provided on Manchester Research Explorer is the Author Accepted Manuscript or Proof version this may differ from the final Published version. If citing, it is advised that you check and use the publisher's definitive version.

\section{General rights}

Copyright and moral rights for the publications made accessible in the Research Explorer are retained by the authors and/or other copyright owners and it is a condition of accessing publications that users recognise and abide by the legal requirements associated with these rights.

\section{Takedown policy}

If you believe that this document breaches copyright please refer to the University of Manchester's Takedown Procedures [http://man.ac.uk/04Y6Bo] or contact uml.scholarlycommunications@manchester.ac.uk providing relevant details, so we can investigate your claim.

\section{OPEN ACCESS}




\title{
Improving Spatial Resolution for EIT Reconstructed Images through Measurement Strategies
}

\author{
Renee K. Y. Chin ${ }^{\# 1}$, Trevor A. York ${ }^{* 2}$ \\ ${ }^{*}$ School of Engineering and Information Technology, Universiti Malaysia Sabah \\ Kota Kinabalu, Sabah, Malaysia \\ reneekychin@ums.edu.my \\ * SISP, School of Electrical and Electronics Engineering, University of Manchester \\ Manchester, United Kingdom \\ t.york@manchester.ac.uk
}

\begin{abstract}
Reconstructed images produced using Electrical Impedance Tomography (EIT) are notorious for their poor spatial resolution. This is largely due to the ill-posed nature of the EIT problem. Much effort has been made in order to improve on spatial resolution of reconstructed images. One of the more direct approaches is by improving the amount of information obtainable. With the development and transition from 2D to 3D tomography, more measurements can be obtained to better inform the inversion problem through expanding the number of electrodes or through measurement strategies. This paper delves into the latter method, providing a comparison study using sensitivity analysis, stability of measurement analysis and spatial resolution analysis, with the aim of providing a better understanding of the influence of varying different parameters on the quality of reconstructed images, before more unconventional methods of increasing information through unconventional measurement strategies are devised.
\end{abstract}

\section{INTRODUCTION}

Electrical Impedance Tomography (EIT) has been widely applied in industrial applications for process imaging for monitoring and control purposes. Its many advantages include ease of application, relatively fast data acquisition time and cost effective. Conventional EIT systems are nonintrusive and non-invasive, whereby the electrodes are confined to the wall of the phantom. While this does not intrude the on-going process that is monitored, it also inevitably limits where measurements can be acquired.

One of the biggest disadvantages of EIT is the quality of the reconstructed images. Tomograms produced using EIT are notorious for having low spatial resolution, especially towards the central region of the image. This is due to the ill-posed nature of the EIT problem. Another contributing factor is due to the restrictiveness of the position of the electrodes.

Much effort has been made to improve the spatial resolution of reconstructed images through increasing the stability of measurements. One of the most commonly used methods is to increase the number of measurements through increasing the number of electrodes or by manipulating the measurement strategy. [1] shows that the obtainable improvement in spatial resolution is ultimately restricted by sensitivity (detectability of changes) and uniqueness of measurements.

This paper consists of six (6) sections. Section II describes the considerations made for the work presented in this paper. Sections III and IV provide the background for the work presented, in terms of modelling and the measurement strategies used. Section V details the results of the comparative studies through sensitivity analysis, stability and uniqueness of measurements and spatial resolution analysis. Conclusion is provided in section VI.

\section{OBTAINING MORE INFORMATION THROUGH MEASUREMENT STRATEGIES}

When planning a measurement strategy, there are several factors that need to be taken into consideration, including measurement stability, number of unique measurements, current injection pattern that can produce sufficient sensitivity across the volume, overall spatial resolution and overall data acquisition time. Spatial resolution is strongly influenced by sensitivity and overall amount of information available for inverse solving.

It has been established that some measurements are likely to be redundant and does not contain unique information for inverse solving, for measurement strategies that include all possible measurements obtainable using a four-terminal measurement strategy. These strategies will also 
result in longer data acquisition time, compromising temporal resolution.

The most commonly used strategy is the adjacent current injection strategy. Depending on the requirement and set-up of applications, other strategies may be preferable. Most industrial process applications adopt the planar electrode arrangement for ease of modeling and computation, as shown in Fig. 1. An electrode arrangement is considered planar even though it is arranged in multiple planes if the electrode planes are treated independently when developing a measurement strategy. There has not been a significant volume of research done on multi-planar strategies to date. Some exceptions include [2], who reported that resolution between planes improved when current injection and voltage measurement are taken between horizontally and vertically adjacent electrodes, and [3] applied cross-planar current drives for 3D imaging. [4] claimed that there were negligible differences between a reconstruction using a planar and a non-planar adjacent measurement strategy. However, the same paper also suggested that there are observable improvements to image quality when current is injected between different planes.

\section{MODELLING}

The data presented in this paper are simulated based on a cylindrical vessel that has 32 wallmounted electrodes, equally-spaced in two planes $(2 \times 16)$ as shown in Fig. 1 . The model is $34 \mathrm{~cm}$ in diameter and $30 \mathrm{~cm}$ in height. The centre of the first plane of electrodes is $10 \mathrm{~cm}$ from the base of the vessel and the height between centres of electrode planes is $10 \mathrm{~cm}$. The 32 electrodes occupy $15 \%$ of the surface area of the model.

Simulations were done using the Electrical Impedance Tomography and Diffuse Optical Tomography Reconstruction Software (EIDORS) [5], hosted by MATLAB. The model is a geometrically accurate Finite Element Method (FEM) model generated using Netgen [6], consisting of more than 12,000 elements. The same model is used for both single-plane and multi-plane simulations.

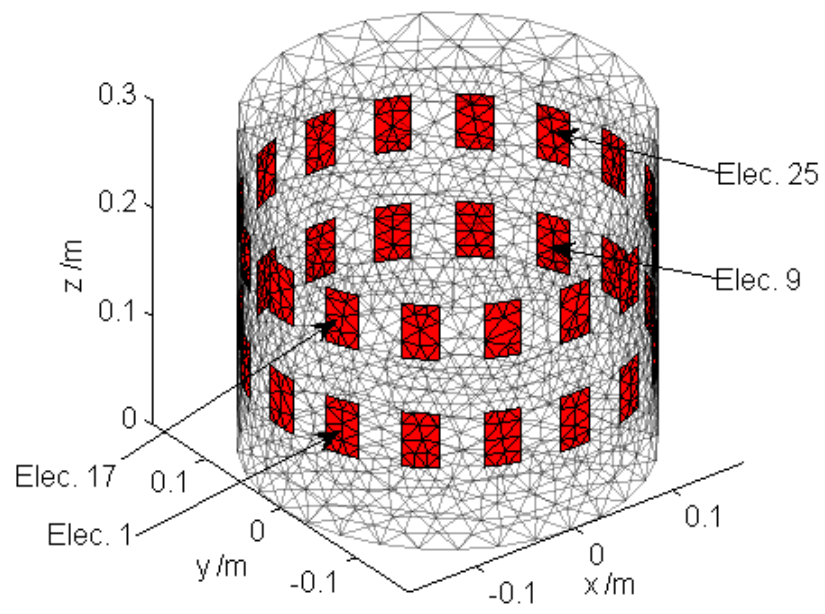

Fig. 1. 3D model of the vessel (electrodes highlighted in red)

IV. MEASUREMENT STRATEGIES

Conventionally, measurement strategies in EIT are all four-terminal measurement protocols [7]. Each current pattern is injected between a pair of electrodes. The most well-established are either adjacent [7] or opposite [8] pairs. All possible horizontally adjacent combinations of electrodes are used to measure differential voltage measurements, with the exception of the current injecting electrodes. All electrodes are used as both current injecting electrodes and measurement electrodes. The functions of the electrodes are interchangeable without any altercation to the measured information.

By definition, $L$ number of electrodes will produce $L(L-3)$ measurements for the adjacent current drive strategy. Meanwhile, the opposite strategy will produce $L(L-4)$ measurements. However, there is the issue where not all measurements are unique. The reciprocity principle is applied to discard redundant measurements; if two pairs of adjacent electrodes used for a particular measurement are interchangeable, the measurements are deemed redundant.

In this paper, strategies with and without the application of reciprocity principle are considered. The benefits of 3D imagine of single-plane verses multi-planar electrode strategies are described in this paper. Both the adjacent and opposite current injection strategies are considered. For multi-planar current injection strategies, horizontally adjacent, vertically adjacent and all possible adjacent combination measurement strategies are compared. 
All results are simulated using the model as shown in Fig. 1. A summary of the current driving and voltage measurement strategies is given in Table I.

TABLE I

Summary of CurRent Driving AND Voltage MEASUREMENT STRATEGIES

\begin{tabular}{|c|c|c|c|}
\hline Strategy & $\begin{array}{l}\text { Current } \\
\text { Injection }\end{array}$ & $\begin{array}{c}\text { Voltage } \\
\text { Measurements }\end{array}$ & $\begin{array}{c}\text { Number of } \\
\text { Measurements }\end{array}$ \\
\hline \multicolumn{4}{|c|}{ Adjacent Strategies } \\
\hline HaPRecip & \multirow{4}{*}{$\begin{array}{l}\text { Horizontally } \\
\text { adjacent }\end{array}$} & $\begin{array}{l}\text { Horizontally adjacent } \\
\text { with reciprocity }\end{array}$ & 208 \\
\hline $\mathrm{HaHa}$ & & Horizontally adjacent & 928 \\
\hline $\mathrm{HaVa}$ & & Vertically adjacent & 448 \\
\hline $\mathrm{HaHVa}$ & & All adjacent & 1376 \\
\hline $\mathrm{VaHa}$ & \multirow{3}{*}{$\begin{array}{l}\text { Vertically } \\
\text { adjacent }\end{array}$} & Horizontally adjacent & 448 \\
\hline $\mathrm{VaVa}$ & & Vertically adjacent & 240 \\
\hline VaHVa & & All adjacent & 688 \\
\hline HVaHVa & All adjacent & All adjacent & 2064 \\
\hline \multicolumn{4}{|c|}{ Opposite Strategies } \\
\hline OPRecip & \multirow{4}{*}{$\begin{array}{l}\text { Opposite on the } \\
\text { the same plane }\end{array}$} & $\begin{array}{l}\text { Horizontally adjacent } \\
\text { with reciprocity }\end{array}$ & 192 \\
\hline $\mathrm{OHa}$ & & Horizontally adjacent & 448 \\
\hline OVa & & Vertically adjacent & 224 \\
\hline OHVa & & All adjacent & 672 \\
\hline O3DHa & \multirow{3}{*}{$\begin{array}{l}\text { Opposite between } \\
\text { different planes }\end{array}$} & Horizontally adjacent & 448 \\
\hline O3DVa & & Vertically adjacent & 224 \\
\hline O3DHVa & & All adjacent & 672 \\
\hline
\end{tabular}

\section{EXPERIMENTAL RESULTS}

Comparative studies are conducted and presented using three tools, namely spectral analysis for measurement stability, sensitivity analysis and spatial resolution analysis.

\section{A. Spectral Analysis}

In EIT, the spectral form is the transformation of the sensitivity matrix (tomographic operator) and the regularisation matrix into a diagonalised form. The image consists of multiple components with varying levels of detail or spatial frequency. The most commonly used numerical technique for spectral analysis in EIT is Singular Value Decomposition (SVD) [9]. Each spectral component within the image is affected by system noise on various degrees; each of the components allows the impact of system noise to influence the image or content to be assessed. As such, SVD is often used to compare system noise and stability of measurements.

Fig. 2 shows a typical example of a set of singular values calculated by applying the adjacent strategy to the HaHa strategy for a 2x16 electrodes model. From Fig. 2, it is shown that after the $433^{\text {th }}$ singular value, the magnitudes of the rest of the singular values are effectively zero to machine precision. This means that although the HaHa strategy is capable of producing 928 measurements, only 433 measurements are unique.

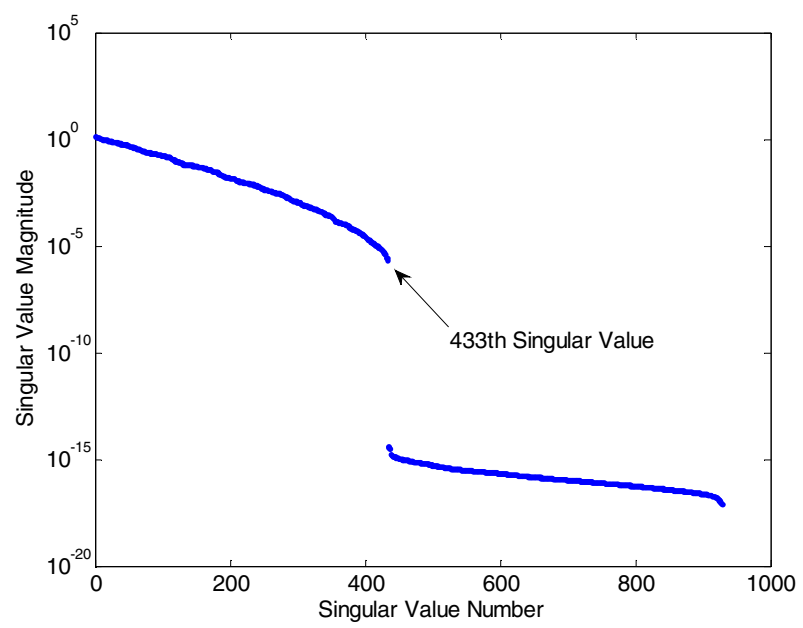

Fig. 2. Logarithmic plot of singular values as ordered in $\Sigma$ for the HAHA strategy (928 measurements) for a 2 x 16 electrodes model

The stability of the measurements can be assessed using the Discrete Picard Condition (DPC) [9] [10]. The measurement is said to be stable if the absolute Fourier coefficients (FCs) decay faster to zero, on average, than the corresponding singular values. [9] suggests a more robust definition based on a moving geometrical mean in place of regular averaging.

Therefore, the DPC is an essential tool when evaluating the noise level on measurements and informs of modelling errors. In practice, measurements contain noise and if a singular component relies on information which has added noise, the information may be unstable and unrecoverable. Fig. 3 shows an example of the application of DPC to evaluate the amount of measurements that is usable for inverse solving for the HaPRecip strategy with $60 \mathrm{~dB}$ Gaussian noise added to the simulated data. The signal-to-noise (SNR) level is in line with the Low-Cost Tomography 2 instrument [11].

Table II shows a summary of unique and stable measurements for various multi-planar strategies. From Table II, it is shown that multi-planar measurements provide additional unique measurements in comparison with single-plane measurement strategies. The lower the percentage of obtainable unique measurements, the less efficient and optimized the strategy. This ultimately 
has an impact on temporal resolution, a limiting factor for dynamic applications. When noise is imposed onto the measurements, the number of stable measurements further decreases the number of usable information for inverse solving.

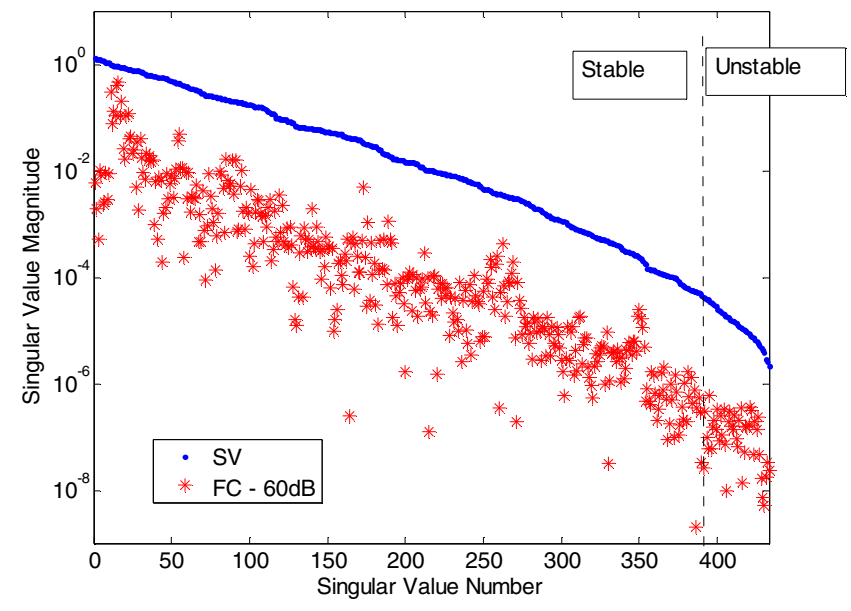

Fig. 3. SVD plot comparing Fourier Coefficients for 60dB Gaussian noise of measurement noise for the HAHA strategy (showing SVs 1 to 433 only)

TABLE II

NUMBER OF UNIQUE MEASUREMENTS OBTAINED FOR DIFFERENT STRATEGIES USING 2-PLANE, 32-ELECTRODES MODEL

\begin{tabular}{|l|c|c|c|}
\hline \multicolumn{1}{|c|}{ Strategy } & $\begin{array}{c}\text { Total Number } \\
\text { of } \\
\text { Measurements }\end{array}$ & $\begin{array}{c}\text { Number of } \\
\text { Unique } \\
\text { Measurements }\end{array}$ & $\begin{array}{c}\text { Number of } \\
\text { Stable } \\
\text { Measurements }\end{array}$ \\
\hline \multicolumn{4}{|c|}{ Adjacent Strategies } \\
\hline HaPRecip & 208 & 208 & 195 \\
HaHa & 928 & 433 & 398 \\
HaVa & 448 & 343 & 313 \\
HaHVa & 1376 & 463 & 437 \\
\hline VaHa & 448 & 343 & 315 \\
VaVa & 240 & 120 & 107 \\
VaHVa & 688 & 344 & 298 \\
\hline HVaHVa & 2064 & 464 & 435 \\
\hline \multicolumn{4}{|c|}{ Opposite Strategies } \\
\hline OPRecip & 192 & 152 & 148 \\
OHa & 448 & 328 & 293 \\
OVa & 224 & 196 & 189 \\
OHVa & 672 & 344 & 319 \\
\hline O3DHa & 448 & 343 & 311 \\
O3DVa & 224 & 196 & 189 \\
O3DHVa & 672 & 344 & 319 \\
\hline
\end{tabular}

\section{B. Sensitivity Analysis}

Sensitivity analysis provides a direct indication of the potential success of a reconstruction algorithm. A detectable sensitivity is the minimum requirement for a successful reconstruction. If a feature if not detectable, it is impossible for any reconstruction algorithm to visualise it. For a discretised model consisting of a finite number of elements, each element has a sensitivity value for any particular measurement. In this paper, the maximum sensitivity of each voxel from a full set of measurement is computed and compared.

Fig. 4 compares the maximum sensitivity magnitude for elements at the height of $0.10 \mathrm{~m}$ from base of vessel for the HaPRecip and OPRecip strategies. It can be seen that the opposite current injection strategy produces higher sensitivity levels in comparison with the adjacent strategy near the centre of the imaged space, as current pattern penetrates across the space for the opposite strategy, while the highest current distribution tends to be highest towards the wall of the vessel when using the adjacent strategy.

Fig. 5 compares the maximum sensitivity magnitude for elements at $0.10 \mathrm{~m}$ from base of model (on-electrode plane) resulting from varying adjacent single and multiple plane current injection strategies. It can be observed that there is little difference between the horizontally adjacent, vertically adjacent and all possible adjacent current injection strategies. Although sensitivity is predominantly influenced by current injection strategy, the benefit of a full-3D current injection strategy $(\mathrm{HVaHa})$ is negligible.

Fig. 6 compares the maximum sensitivity magnitude for on- and off-electrode plane for the $\mathrm{HaHa}, \mathrm{VaHa}$ and $\mathrm{HVaHa}$ strategies. The results show that there is noticeable improvement of maximum level of sensitivity on the off-electrode planes towards the top and bottom of the vessel with the $\mathrm{HVaHa}$ strategy providing the best sensitivity levels out of the three adjacent current injection strategies compared.

\section{Spatial Resolution Analysis}

Spatial resolution analysis makes use of the resolution matrix which contains information about the correlation between elements in a discretised model. The technique used in this paper is based on the technique that was first proposed by [12], which was adapted from the work by [13].

In spatial resolution analysis, the Full-Width Half-Maximum (FWHM) technique [14] is used. FWHM is the measure of the distance between two points in an image that share the same solution value that is half the peak value. The lower the percentage of FWHM, the better the spatial resolution. 


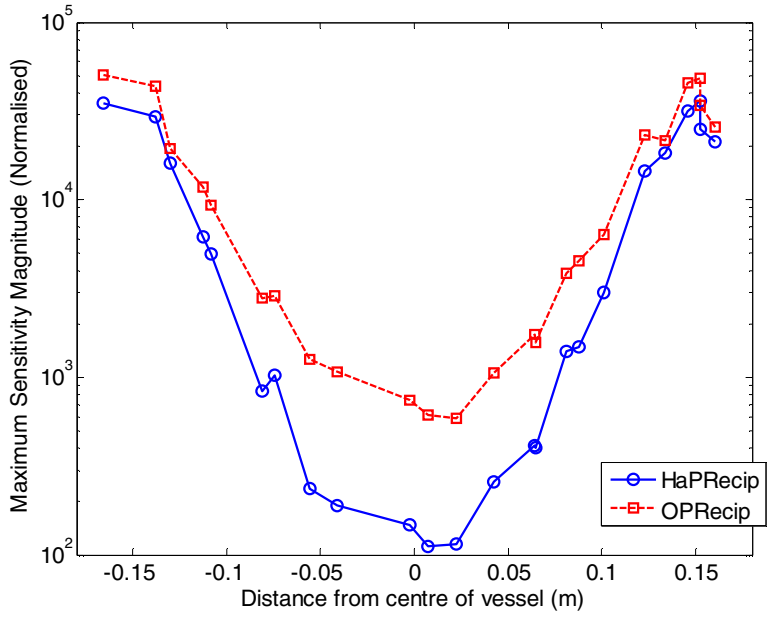

Fig. 4. Sensitivity analysis comparing HaPREcip and OPRecip at electrode plane (height $=0.10 \mathrm{~m}$ from base of vessel)

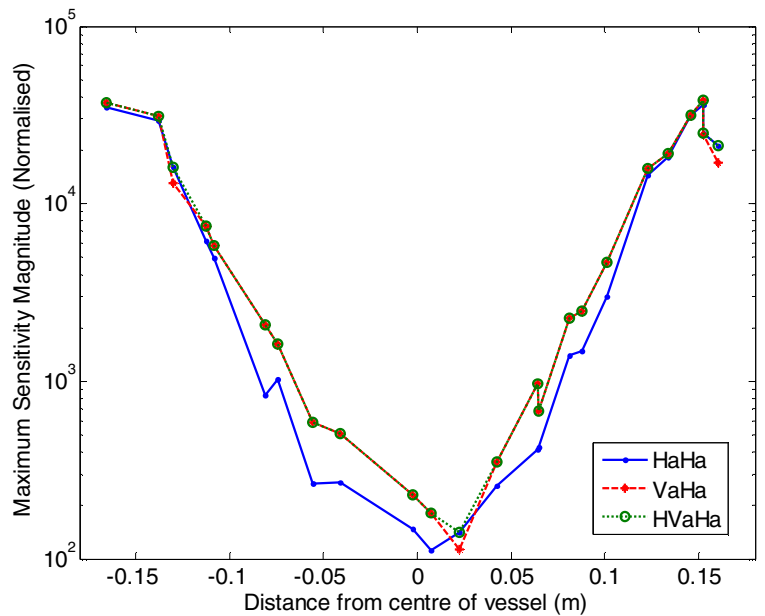

Fig. 5. Sensitivity analysis comparing single and multiple plane adjacent current injection strategy at electrode plane (at $0.10 \mathrm{~m}$ from base of vessel)

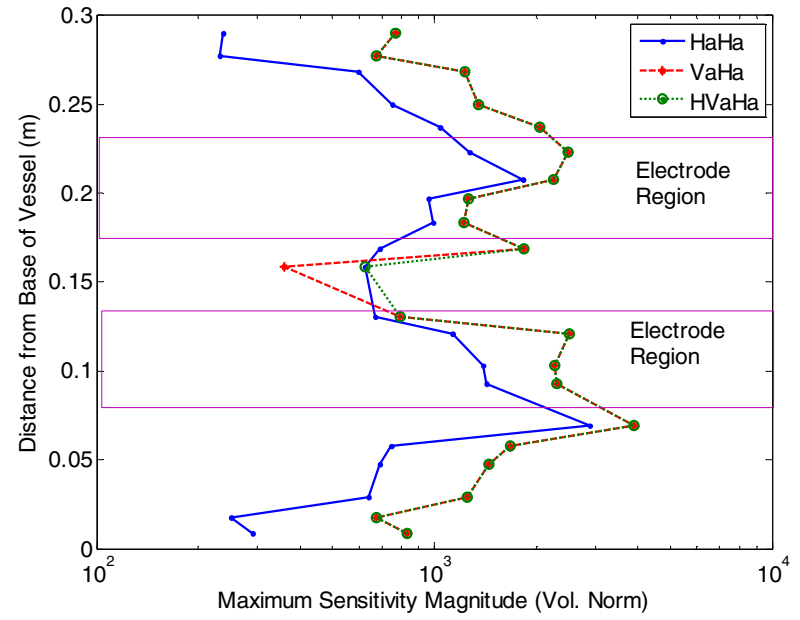

Fig. 6. Sensitivity analysis comparing single and multiple plane adjacent current injection strategy at various heights at equidistance between wall and centre of vessel

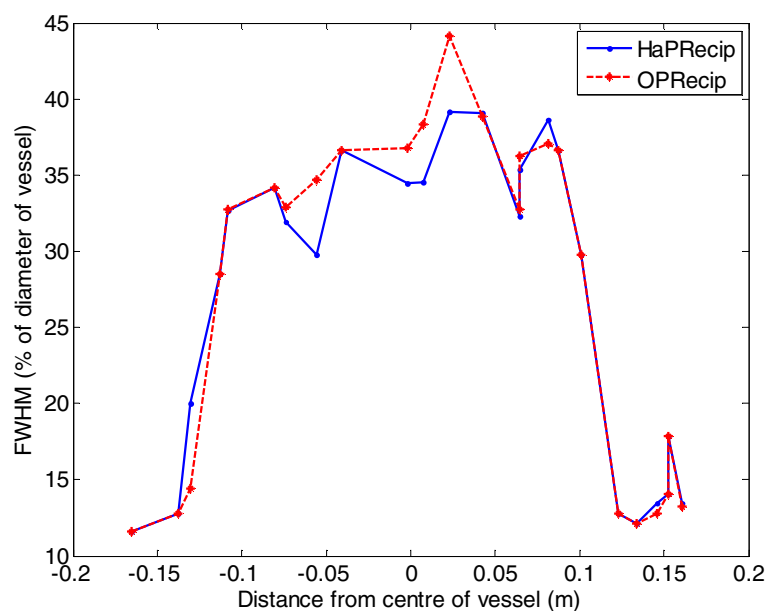

Fig. 7. Spatial resolution analysis comparing HaPRecip and OPRecip strategies at $0.10 \mathrm{~m}$ from base of vessel

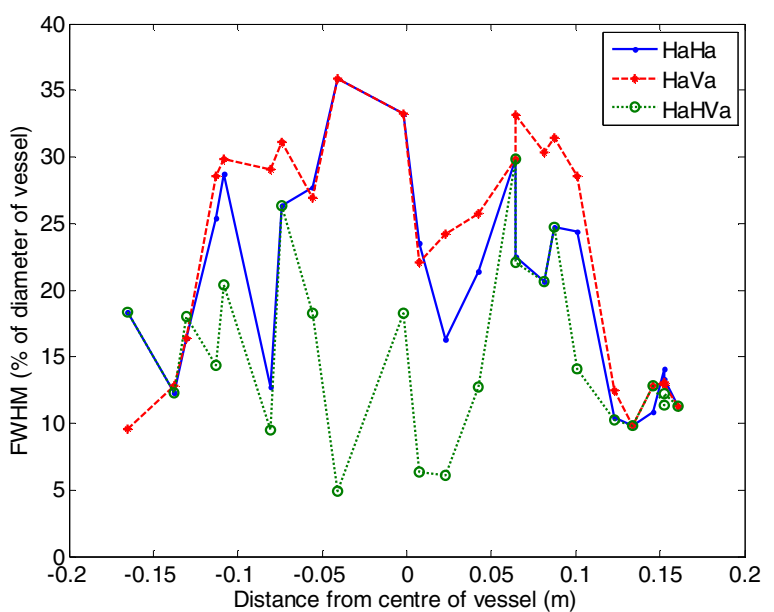

Fig. 8. Spatial resolution analysis comparing single and multi-plane voltage measurement strategies for adjacent current injection strategy at $0.10 \mathrm{~m}$ from base of vessel

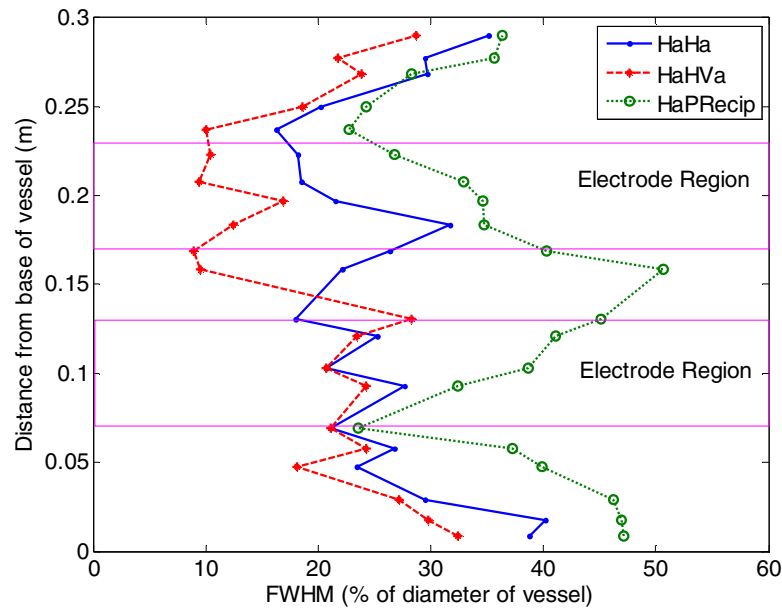

Fig. 9 Spatial resolution analysis comparing single and multi-plane measurement strategy at various heights at equidistance between wall and centre of vessel 
Fig. 7 compares the spatial resolution between the HaPRecip and OPRecip strategies. The result observed in Fig. 7 is not as anticipated, considering the significant difference in sensitivity level as shown in Fig. 4. It is worth noting that both strategies considered produce similar amount of usable information for reconstruction. This is likely to be the reason why the spatial resolutions are evenly matched across the plane for both strategies, as spatial resolution is largely influenced by the amount of usable content available for reconstruction.

Fig. 8 compares the single and multi-plane measurement strategies for adjacent current driving strategies. The results indicate that there is negligible difference in spatial resolution across the plane. Arguably, the HVaHa strategy produces an overall better FWHM across the plane.

Fig. 9 illustrates the benefit of multi-plane measurement strategy. The difference is especially prominent for the off-plane region between the two electrode planes. This may be due to the abundance of information acquired through the full-3D measurements. Interestingly, the spatial resolution is not necessarily the best within the electrode regions.

\section{CONCLUSION}

The results presented in this paper indicates that while measurement strategies are capable of increasing the number of measurements from a fixed set of electrodes configuration, the extra measurements acquired may not be unique, and therefore the strategy is deemed not optimised.

Comparing the adjacent and opposite current drive strategies, while the sensitivity analysis clearly indicates that the opposite current injection strategy produces superior level of maximum sensitivity, the spatial resolution analysis shows that there is negligible improvement and therefore the quality of the reconstructed images would bear insignificant improvement.

Comparing single and multi-plane measurement strategies, the results indicate that there are visible benefits in acquiring multi-plane measurements, although the difference is not prominent.

The results indicate that that the sensitivity (detectability) is predominantly influenced by the current injection strategy, while spatial resolution (visibility or reconstructability) is largely influenced by the measurement strategy and the resultant number of measurements available for image reconstruction.

The results in this paper hint that the location of electrodes plays an important role in improving spatial resolution of reconstructed images. Logically, sensitivity and spatial resolution are better for elements within close proximity of the electrodes. The results also indicate that beyond a point, the increase of information through this method reaches saturation. This means no further improvement can be achieved.

\section{ACKNOWLEDGMENT}

The authors would like to thank Dr. Stephen C. Murphy for his contribution and support of the work presented in this paper.

\section{REFERENCES}

[1] N. Poloydorides and H. McCann, "Electrode configuration for improved spatial resolution in Electrical Impedance Tomography," Meas. Sci. Technol., vol. 13, pp. 1862-1870, 2002.

[2] P. Metherall, D.C. Barber, R.H. Smallwood and B.H. Brown, "Threedimensional Electrical Impedance Tomography," Nature, vol. 380, pp. 509-512, 1996

[3] L.M. Heikkinen, J. Kourunen, J. Rastas, T. Savolainen, P.J. Vauhkonen, J.P. Kaipio and M. Vauhkonen, "Real time threedimensional Electrical Impedance Tomography applied in multiphase flow imaging," in Proc. $4^{\text {th }}$ WCIPT '05, 2005, pp. 540-545.

[4] D.R. Stephenson, J.L. Davidson, W.R.B. Lionheart, B.D. Grieve and T. York, "Comparison of 3D image reconstruction techniques using real Electrical Impedance measurement data," in Proc. $4^{\text {th }}$ WCIPT '05, 2005, pp. 643-650.

[5] N. Polydorides and W.R.B. Lionheart, "A Matlab toolkit for threedimensional Eletrical Impedance Tomography: A contribution to the Electrical Impedance and Diffuse Optical Reconstruction Software project," Meas. Sci. Technol., vol. 13, pp. 1871-1883, 2002.

[6] J. Schoberl. (2008) Netgen: Automatic Mesh Generator. Available: http://www.hpfem.jku.at/netgen

[7] D.C. Barber and B.H. Brown, "Applied potential tomography," J. Phys. E: Sci. Instrum., vol. 17, pp. 723-33, 1984.

[8] J.G. Webster, Ed., Electrical Impedance Tomography, IOP: Bristol, 1990

[9] P.C. Hansen, Rank-Deficient and Discrete Ill-Posed Problems: Numerical Aspects of Linear Inversion, SIAM: Philadelphia, 1998.

[10] L. Wu, "A parameter choice method for Tikhonov regularization," Elec. Trans on Numerical Analysis, vol. 16, pp. 107-128, 2003.

[11] T.A. York, S. Murphy, A. Burnett-Thompson and B. Grieve, "An accessible Electrical Impedance Imaging System," in Proc. $4^{\text {th }}$ WCIPT '05, 2005, pp. 100-105.

[12] S.C. Murphy, Ad Hoc Electrode Arrangement for Electrical Tomography, Ph.D Thesis, University of Manchester, United Kingdom, 2008

[13] W. Menke, Geophysical Data Analysis: Discrete Inverse Theory (Rev. Ed.), Academic Press: San Diego, 1989.

[14] J.L. Wheeler, W. Wang and M. Tang, Á comparison of methods for measurement of spatial resolution in two-dimensional circular EIT images," Physiol. Meas., vol. 23, pp. 169-175, 2002. 